\title{
An Immersive Virtual Reality Platform Integrating Human ECOG \& sEEG: Implementation \& Noise Analysis
}

This paper was downloaded from TechRxiv (https://www.techrxiv.org).

\section{LICENSE}

CC BY-NC-SA 4.0

SUBMISSION DATE / POSTED DATE

31-01-2022 / 03-02-2022

\section{CITATION}

Paschall, Courtnie; Rao, Rajesh P.N.; Hauptman, Jason; G. Ojemann, Jeffrey; Herron, Jeffrey (2022): An Immersive Virtual Reality Platform Integrating Human ECOG \& sEEG: Implementation \& Noise Analysis. TechRxiv. Preprint. https://doi.org/10.36227/techrxiv.19099772.v1

$\mathrm{DOI}$ 


\title{
An Immersive Virtual Reality Platform Integrating Human ECOG \& sEEG: Implementation \& Noise Analysis
}

Courtnie J. Paschall, Member, IEEE, Rajesh P.N. Rao, Senior Member, IEEE, Jason Hauptmann, Jeffrey Ojemann, and Jeffrey Herron, Member, IEEE
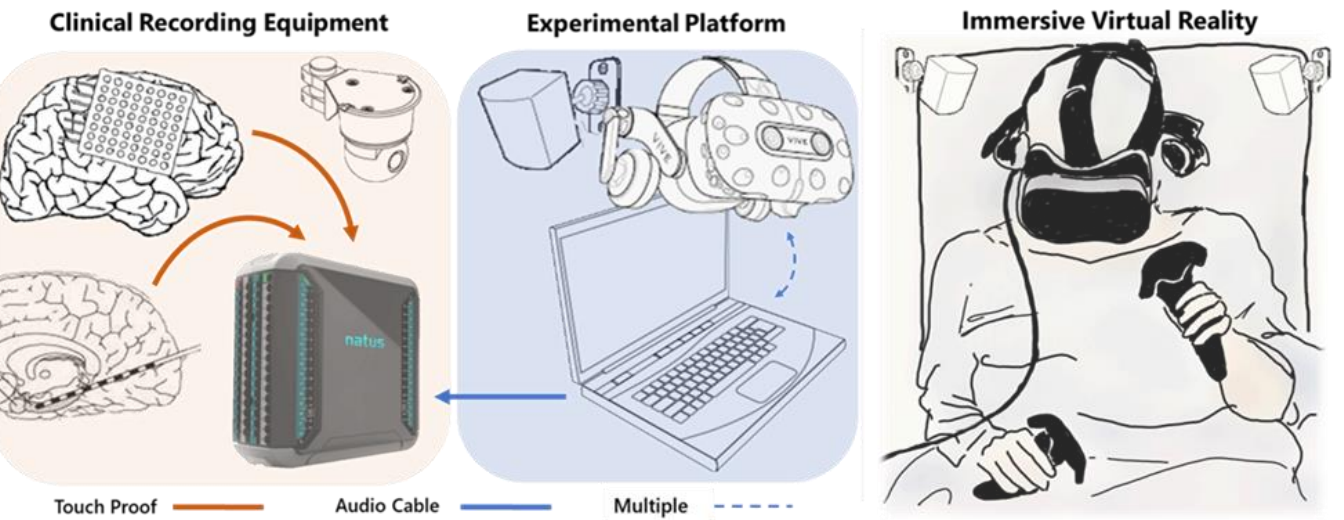

Fig. 1 System diagram. (left) Clinical recording hardware includes implanted electrodes, Natus Quantum amplifier and clinical neural recording suite, and in-room cameras recording continuous video and audio data. (middle) The experimental VR platform includes a VRready gaming laptop, HTC Vive Pro Eye headset, Vive controllers 2.0, and SteamVR base stations 2.0. (right) A patient immersed in a VR task.

\begin{abstract}
Virtual Reality (VR) offers a powerful new platform for experimental neuroscience that has become increasingly popular with non-invasive neural recording modalities like electroencephalography (EEG) and functional MRI. Despite this broad appeal, no analysis yet exists quantifying the impact of concurrent VR use on the fidelity of electrophysiological signal features acquired intracranially. In this work, we evaluate a VR platform for behavioral experimentation with in-patient human subjects undergoing invasive neural recording with electrocorticography (ECOG) and stereoelectroencephalography (sEEG). We present a noise analysis of data from two subjects, one child and one adult, both VR naïve prior to our experimental sessions. We demonstrate a line noise increase due to VR hardware that is effectively mitigated by common average rereferencing and no significant increase of the highfrequency noise floor between VR headset on and off conditions. We also discuss donning and removal of the VR headset with this patient population, as well as tips for addressing VR hesitancy and first immersion. This work demonstrates the feasibility of immersive VR experimentation with awake intracranial patients and is, to our knowledge, the first demonstration of its kind.
\end{abstract}

Index Terms-Behavioral neuroscience, neural engineering, ECOG, sEEG, brain-computer interface (BCI)

\section{INTRODUCTION}

In immersive virtual reality (VR), a worn headset, or Head Mounted Display (HMD), presents high-resolution images with wide field of view, independently to each eye. This binocular presentation allows for perception of depth and parallax, creating an immersive

- Courtnie J. Paschall, Rajesh P.N. Rao, Jeffrey Ojemann and Jeffrey Herron are with the University of Washington. E-mails: \{copa2894 | rpnr | jeffherr |jojemann\}@uw.edu

- Kurt Weaver is with the University of Washington School of Medicine.Email: weaverk@uw.edu.

- Jason Hauptmann is with Seattle Children's Hospital. Email: Jason.Hauptman@seattlechildrens.org

Manuscript received xx xxx. 201x; accepted xx xxx. are01x. Date of Publication $x x$ xxx. 201x; date of current version $x x x x x$. 201x. For information on obtaining reprints of this article, please send e-mail to: reprints@ieee.org .

Digital Object Identifier: xx.xxxx/TVCG.201x.xxxxxxx/. environment that feels visually real and expansive. Modern VR headsets allow for precise tracking and visual rendering of headset movement and hand-held controller positioning which are used to build a first-person avatar in VR that precisely follows a user's head and hand movements. The effect is an interactive, alternate reality that is designed, monitored, and controlled by the VR developer. For human cognitive and behavioral neuroscience, this offers a new experimental platform with unprecedented control over a wide range of environmental parameters that include object dynamics, physics, body rendering, and the underlying distributions that govern all probabilistic interactions.

Immersive VR platforms have significantly contributed to animal model research for decades, though only recently have technological advancements made immersive virtual reality accessible in human studies. Portable and immersive VR has been successfully paired with non-invasive electroencephalography (EEG) to explore the neural correlates of human behavior, with particular emphasis on embodiment and embodied memory. VR-EEG studies are also 
contributing to next-generation functional brain computer interface (BCI) $[1,2]$

While VR-BCI research with non-invasive scalp electrodes is underway, a virtual reality platform for the in-patient, invasive intracranial electrode population had not yet been implemented. This is an important next step for VR-integrated human behavioral and neuroscience experiments, as intracranial recordings sample directly from cortical and subcortical brain structures with significant improvements in spatial and temporal signal resolution compared to EEG. This capacity is an important consideration for the design and efficacy of bidirectional brain computer interfaces (BCIs), as ECOG and sEEG electrodes also enable brain stimulation directly into a desired structural target [3].

To advance VR technology into the invasive clinical recording space, we present the first critical assessment of VR-HMDs during clinical neural recordings. We also delineate a safe and comfortable procedure for donning and doffing the headset and address latent concerns about immersive experiments with VR naïve subjects. To characterize the impact of the headset on electro-physiology data acquisition and signal features, we acquired neural activity recordings from two subjects while the VR headset was on and with the headset off. Noise analyses are completed as well as other exploratory analysis (e.g regression of noise with electrode depth). We also demonstrate signal fidelity during headset positioning and discuss techniques engagement with virtual reality hardware.

VR offers unparalleled experimental control for enhancing existing experimental protocols and opens exciting new directions human neuroscience research. This work is intended to answer basic questions of efficacy and reliability as the intracranial neural recording field moves to embrace virtual reality task design.

\section{EXPOSITION}

Clinical data were recorded in an approved IRB protocol following formal consent of the subject and legal guardian if under 18 years of age. Virtual reality headsets are believed to present no additional risk to the patient. Data were collected from two subjects implanted with intracranial electrodes for seizure localization. Subject 1 was a 16 year-old male with a prior left temporal lobectomy, implanted with 24 left temporal ECOG and 90 left hemisphere sEEG electrodes (114 in total, see Fig. 2 left). Subject 1 remained in virtualis re for over 26 minutes in one continuous session. Subject 2 was a 20 -year-old female implanted with 142 left hemisphere sEEG electrodes (see Fig. 2,

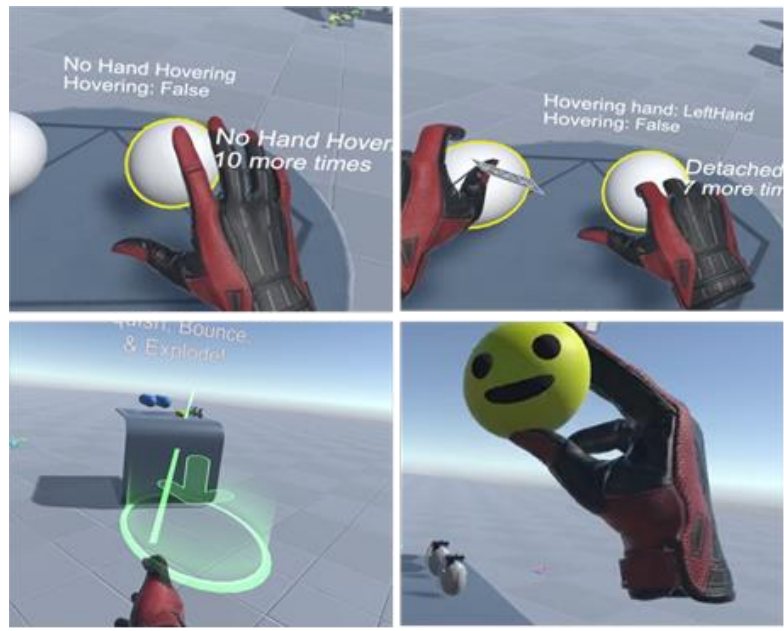

Fig. 3. Example images from an experimental session with Subject 2. This task involves virtual object interaction.

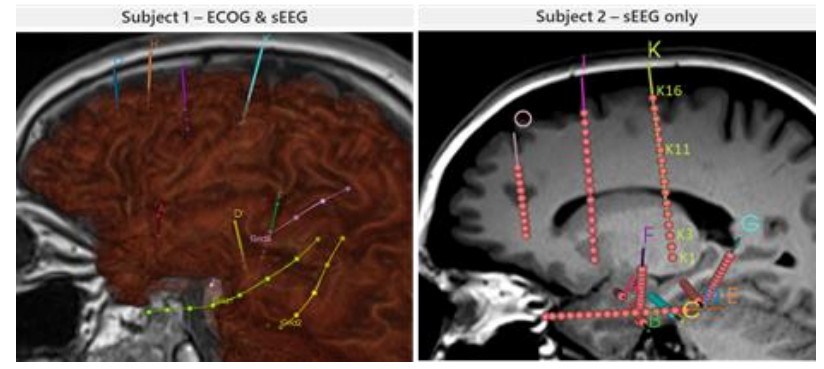

Fig. 2. Electrode localization images for Subjects 1 and 2. Subject 1 was implanted with both cortical and subcortical (ECOG and sEEG) electrodes, and Subject 2 subcortical (sEEG) only.

right). Subject 2 spent 68 minutes in VR across three sessions over two days (12 minutes on the first and 56 minutes on the second day). Subject 1 of this study is, as far as the we are aware, the first in-patient subject to don an immersive virtual reality headset while undergoing invasive neural recording.

\subsection{Clinical Recording System \& Electrode Localization}

Recorded clinical data included continuous intracranial ECOG and sEEG neural signals, in-room video from two wall-mounted cameras, in-room sound, and two DC input channels recording a stereo audio synchronization signal from the task VR laptop (See Fig. 1).

Neural signals were recorded by the Natus Quantum biosignal acquisition system (Natus Medical Incorporated, San Carlos, CA) at a sampling rate of $1024 \mathrm{~Hz}$, with individual contacts referenced to screw electrodes physically anchoring each implanted probe. The two DC input channels were also recorded at $1024 \mathrm{~Hz}$, referenced to electrical ground at recording, and later bipolar referenced to remove DC bias in the clinically recorded audio signal. The audio signal was used for temporal alignment of the independently recorded clinical and VR task data. Limited discussion of behaviorally aligned data is included here.

In-room video was captured at $30 \mathrm{fps}$ from two orthogonal views of the patient. This is recorded for clinical purposes to capture seizure, preictal, and postictal behavior. Research analysis used the video capture for initial approximate behavioral alignment and labelling of patient behavior outside of the recorded task variables (e.g. verbal responses).

For electrode localization, preoperative magnetic resonance Imaging (MRI) data were acquired on a clinical Philips 3T Achieva scanner using a standard 8 channel SENSE head coil, and postoperative computerized tomography (CT) scans were acquired on a CereTom scanner $(512 \times 512 \times 88$ matrix resulting in an in-plane resolution of $.5 \times .5 \mathrm{~mm} / 1.25$ slice thickness). The preoperative $\mathrm{T} 1$ MRI was co-registered with post-operative CT scans using an affine registration through Statistical Parametric Mapping (SPM) software package (https://www.fil.ion.uclac.uk/spm/doc/). Three dimensional reconstructions of the pial surface were generated using Freesurfer (http://surfer.nmr.mgh.harvard.edu/). Electrode channel positions were estimated from postoperative $\mathrm{CT}$ and projected to the reconstructed pial surface (ECOG) or subcortex (sEEG) (see Fig. 2). A secondary registration into MNI152 $1 \mathrm{~mm}$ space enabled automated atlas identification of each electrode.

\subsection{Virtual Reality Experimental Platform}

Our VR platform utilizes a VR-ready laptop with a high-end gaming GPU and a wired HTC Vive Pro Eye head-mounted display (HMD). 


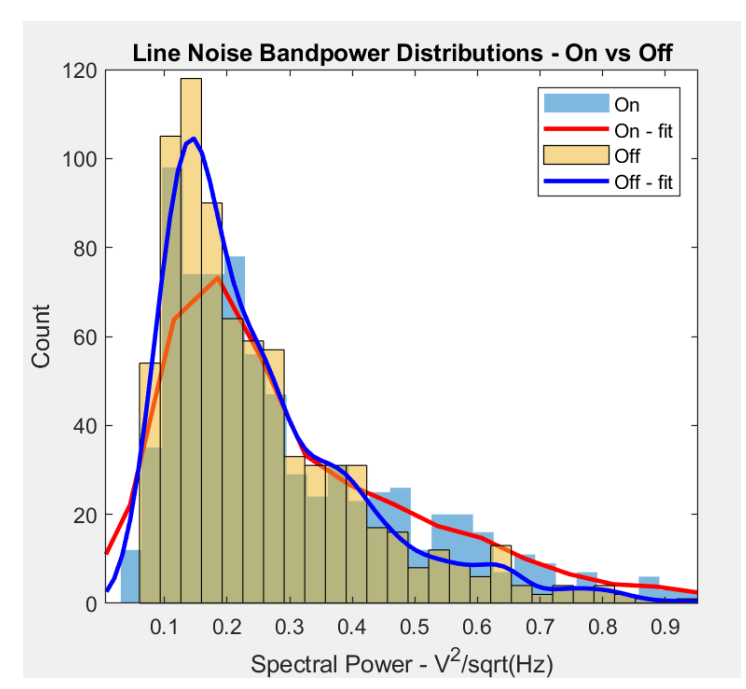

Fig. 4. Distribution of line noise $(57-62 \mathrm{~Hz})$ bandpower values for Subject 1 during 22min of neural recording with the VR headset on (blue bars) and matched duration of neural recording with the headset off (yellow bars). Distributions were estimated using kernel method for the on (red line) and off (blue line) conditions and the distance between the distributions calculated as the symmetric JensenShannon Divergence (JSD).

This HMD boasts a $90 \mathrm{~Hz}$ refresh rate on high pixel density, binocular screens for a high-definition, low latency, immersive virtual reality experience. This system was specifically selected to reduce the chance of virtual reality sickness (VRS) in our subjects. To avoid VRS, we also designed our tasks to have simple, neutral backdrops with infinite horizons and minimal requirement for independent head movement $[4,5]$.

The HTC Vive Pro uses two base stations for submillimeter controller and HMD tracking. For use in the hospital, base stations are mounted on extensible tripods and positioned a few feet from the patient's bed to ensure base station tracking coverage of all potential dynamic movements during the VR tasks (see Fig. 1). Controllers are presented to the patients prior to VR immersion to develop familiarity with the trigger, grip, and trackpad inputs.

The VR headset was donned and removed half a dozen times, without discomfort in either of the two patients presented here. To put the headset over the subject's headwrap, first extend the VR headset to its maximum settings. Then, have the patient hold the HMD display against their face in the most comfortable position for them, while the researcher positions the head strap, mindful of the "braid" of electrode cables gathered at the back of the patient's head. Finally, guide the subject's hands so they may to tighten the headset for themselves, noting that secure headsets are more comfortable over time than loose ones. Both subjects in this study reported ease and comfort with the donning procedure and wearing of the headset for extended periods of time (collectively, over an hour). We noticed that both subjects tightened their own headsets beyond what we initially expected, creating a better and more secure fit. Moreover, both subjects opted to remain in VR when given the choice to either explore additional VR experiences (e.g. Google Earth) or remove the headset. Specifically, following experimental data collection, Subject 1 elected to explore a SteamVR mountain top lodge scene and Subject 2 explored Paris, Tokyo, and Volcanos National Park, HI in Google Earth VR.

Neither Subject 1 nor Subject 2 had prior experience with immersive virtual reality and adapted to the VR tasks readily and neither reported discomfort during their virtual reality sessions. The experience was well tolerated by both subjects with neither reporting an adverse event. As researchers, however, we noted that although both subjects reported no discomfort or disorientation during or after their time in VR, they each spent a few seconds visibly "collecting themselves" (blinking, staring forward, looking at their hands) after their longest VR sessions. This shared behavior may suggest a sensory remapping following the genuine, experiential immersion that modern VR headsets and tracked controllers provide. Example images from an experimental session with Subject 2 are provided for context (See Fig. 3).

\subsection{Noise Analysis}

For both subjects, concurrent in-room clinical video was used to define epochs of time during which the headset was on, off, being put on, or being removed. In Subject 1, 22 continuous minutes of neural recording during which the headset was on were identified, as well as 22 minutes with the headset off. In Subject 2, two separate headset-on epochs of 4.75 minutes and 7.23 minutes were identified. These epochs were separated by 23 minutes of headset-off neural recording. Thus, in Subject 2, continuous neural recording while donning or removing the headset was identified enabling visualization of the impact of the HMD donning and removal process on the clinical recording. As with Subject 1, matched headset-off epochs were identified for analysis such that HMD on and off durations were equal.

\subsubsection{Signals Preprocessing}

Data were minimally pre-processed to preserve recorded noise. In Subject 1, two channels were removed from subsequent analysis due to large variations of amplitude magnitude in both the on and off conditions, in excess of 4 standard deviations. It is a standard preprocessing step to remove from analysis volatile recording channels that may be broken, not implanted in neural tissue, or otherwise not recording reliable neural signal [6]. Two additional

\begin{tabular}{|c|c|c|}
\hline Subject 1 & $57-62 \mathrm{~Hz}$ & $125-240 \mathrm{~Hz}$ \\
\hline $\begin{array}{l}\text { Average Noise On } \\
\text { (CAR On) }\end{array}$ & $0.318(0.157)$ & $0.0199(0.0074)$ \\
\hline $\begin{array}{l}\text { Average Noise Off } \\
\text { (CAR Off) }\end{array}$ & $0.251(0.154)$ & $0.0342(0.0085)$ \\
\hline Ratio On/Off & $1.2663(1.017)$ & $0.583(0.871)$ \\
\hline JSD & $4.482(0.432)$ & $14.436(0.861)$ \\
\hline JSD sEEG \& ECOG & $\begin{array}{l}2.933(0.351) \\
\& 1.549(0.081)\end{array}$ & $\begin{array}{l}11.6469(0.5020) \\
\& 2.7893(0.3592)\end{array}$ \\
\hline Shuffled Range & $19.224-23.989$ & $21.3729-22.5012$ \\
\hline Bootstrap Range & $1.504-4.3845$ & $13.6936-14.6712$ \\
\hline Subject 2 & $57-62 \mathrm{~Hz}$ & $125-240 \mathrm{~Hz}$ \\
\hline $\begin{array}{l}\text { Average Noise On } \\
\text { (CAR On) }\end{array}$ & $\begin{array}{l}1.886(0.260) \\
1.486(0.290)\end{array}$ & $\begin{array}{l}0.082(0.011) \\
0.033(0.010)\end{array}$ \\
\hline $\begin{array}{l}\text { Average Noise Off } \\
\text { (CAR Off) }\end{array}$ & $\begin{array}{l}1.854(0.273) \\
1.500(0.256)\end{array}$ & $\begin{array}{l}0.023(0.006) \\
0.032(0.008)\end{array}$ \\
\hline Ratio On/Off & $\begin{array}{l}1.007(0.954) \\
0.991(1.133)\end{array}$ & $\begin{array}{l}0.560(1.712) \\
1.057(1.317)\end{array}$ \\
\hline JSD & $\begin{array}{l}28.617(0.923) \\
2.547(2.872)\end{array}$ & $\begin{array}{l}156.2(8.960) \\
4.660(3.634)\end{array}$ \\
\hline Shuffled Range & $\begin{array}{l}409.547-512.516 \\
372.102-456.937\end{array}$ & $\begin{array}{l}119.192-125.010 \\
54.511-57.253\end{array}$ \\
\hline Bootstrap Range & $\begin{array}{r}29.146-71.386 \\
25.686-60.484\end{array}$ & $\begin{array}{l}0.830-1.468 \\
0.126-0.684\end{array}$ \\
\hline
\end{tabular}

Table 2. Bandpower calculations for subjects 1 and 2 are listed here, for both line noise (57-62) and noise floor (125-240) bands. In parenthesis beside each value is the result of the same calculation on signals preprocessed by common average rereferencing (CAR). As two headset-on epochs were identified for analysis in Subject 2, two sets of data are presented. 
channels were removed: the first for line noise $(57-62 \mathrm{~Hz})$ band power in excess of 4 standard deviations compared to other channels in both the headset on and off conditions, and the second for broadband noise power in excess of 6 standard deviations in only the headset-on condition. This channel was a cortical ECoG electrode localized to the left frontal pole. Visual inspection of this channel's raw signal showed it to be quite noisy in both the on and off conditions, although the noise power changed markedly between conditions. It is unclear why this channel was particularly susceptible to noise in the headset on condition. Noise analysis in Subject 1 proceeded with signals recorded from 110 of the 114 electrodes.

In Subject 2, all sEEG channels demonstrated similar amplitude variances and although some channels displayed susceptibility to line noise bandpower increases in the headset-on condition, the noise floor remained comparable to headset-off conditions and nearby electrodes. Data from all electrodes were kept for analysis.

Neural signals data were analysed as both "raw signals," the local field potential (LFP) recordings referenced to a scalp needle electrode and grounded to a skull screw, and as common average rereferenced (CAR) signals. In CAR, each channel was referenced to the mean signal of all electrodes of a similar type within each patient. In Subject 1, all subcortical sEEG channels were common average rereferenced to each other, independently of the ECOG channels, which were rereferenced to the average of their own signals. Cortical and sEEG channels have different impedance profiles and thus ought to be rereferenced independently. In our data, cortical channels had an average of 2.41 times the line noise power of sEEG channels in the headset on condition, and 1.54 times the line noise power in the headset off condition. These ECOG vs sEEG ratios did not change greatly after independent rereferencing (2.99 and 1.61) but plummeted to 0.01 and 0.02 after non-independent rereferencing. This suggests that improper CAR may redistribute the increased noise power in ECOG onto the sEEG recordings.

\subsubsection{Welch's Method for Broadband Power Spectral Density}

To evaluate the spectral effect of wearing the HMD, Welch method power spectral density (PSD) estimates were calculated in $1 \mathrm{~Hz}$ frequency steps from 2 to $240 \mathrm{~Hz}$ ( 1 second Hamming windows, $50 \%$ overlap, see Fig. 5). In both frequency bands, bandpower was calculated using an area-under-the-curve estimate by rectangular method from the PSDs of each electrode. The width of the $60 \mathrm{~Hz}$ line noise peak was seen to include frequencies $57-63 \mathrm{~Hz}$ on the PSDs, setting the frequency range for line noise analysis. A qualitative change in high-frequency broadband power was evident between headset on and headset off raw signal PSDs. Thus a separate "noise floor" estimate was also assessed for the high-frequency range (125$240 \mathrm{~Hz})$.

As seen in Fig. 4, CAR demonstrates robust line noise removal $(60 \mathrm{~Hz}$ and calculated harmonics) between the headset on and headset off conditions in both subjects. The actual line noise $(57-62 \mathrm{~Hz})$ and high-frequency broadband noise $(125-240 \mathrm{~Hz})$ mean power estimates in the headset on and off conditions, both before and after common average referencing, are listed in Table 1 . This table also lists the ratio between headset on and off bandpower as well as results from the distribution divergence metric, symmetric Jensen-Shannon Divergence (JSD).

The spectral power distributions of these noise bands were determined to be non-normal by chi-squared goodness of fit and Anderson-Darling tests, the distributions of each on-vs-off and rawvs-car condition were fit suing a kernel method and assessed by chisquared goodness of fit. The distance between each headset on and headset off distribution was calculated using the JSD metric, where lower JSD values indicate less distance between distributions. Two randomization schemes were implemented to help evaluate the significance of the resultant JSD metrics. Additionally, a permutationbased statistical approach was applied in which headset on vs off labels were randomly shuffled, and the JSD recalculated each time to create a "null" distribution of JSD values. In a second randomization approach, 100 subsamples of each of the on and off bandpower estimates were individually compared, thus bootstrapping a windowed JSD metric to give context to the overall JSD.

Bandpower distributions were visualized with histograms and cumulative distribution plots, and then characterized by nonparametric kernel fit of the distribution (See Fig. 5). A symmetric evaluation of the distance between the on vs. off distributions, the Jensen-Shannon Divergence metric was calculated between headset on and off bandpower distributions per patient, per noise band of interest (line noise or noise floor), and for raw or CAR preprocessed data. Table 1 lists the results of the JSD calculations. For example, in

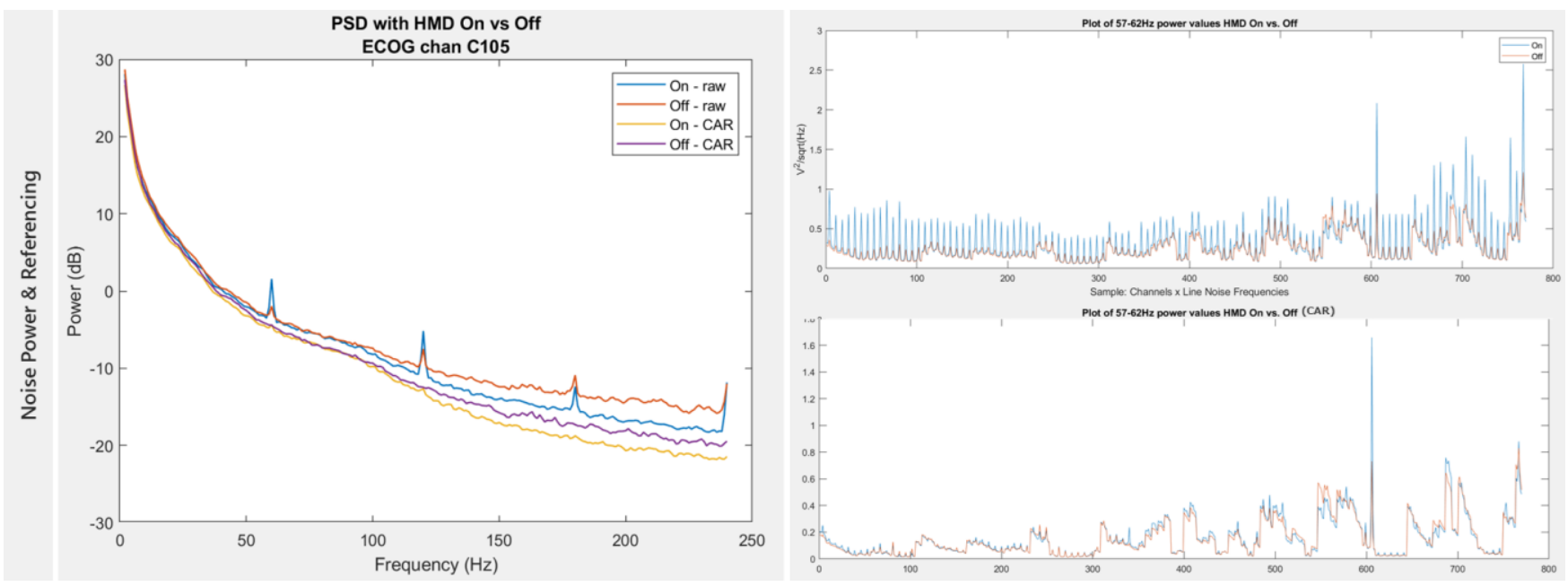

Fig. 5. (left) The Welch power spectral density plot overlays the Welch PSDs of both raw and CAR signals in the headset-on and headset-off conditions for an example electrode in Subject 2. Note the successful removal of in-phase line noise with simple common-average rereferencing. (right) The power values of neural signals across all channels with the headset on (blue) and off (orange) are compared, with the top plot depicting the raw signals and the bottom plot showing the same signals after CAR. The on-condition signals prior to CAR demonstrate a larger line noise bandpower (right top, blue) over the headset-off condition, though this strong visual difference disappears after the rereferencing (right bottom). 
Subject 1, the JSD metric for line noise bandpower between on and off conditions was 4.4820 (for raw signal) and 0.4318 (for CAR signal).

In 1000 randomized shufflings of on-off labels, the range of the resultant JSDs was $19.22-23.99$ (Subject 1, line noise, raw), and in 1000 bootstrapped comparisons, the range was $1.5040-4.3845$. For the shuffled randomization result, the real JSD was much less than the null distribution range $(4.4820<$ [19.22 23.99]), suggesting that the real on and off distributions were much closer than distances between 1000 random distributions generated from the on-off bandpower data. The bootstrapped randomization statistics $(4.4820>$ [1.5040 4.3845]) indicate that the SJD metric is stable: that the calculated SJD fairly represents, though narrowly overestimates, the range of SJD values inherent within the bandpower data. The calculated JSD values and ranges are provided in Table 1, as are JSD values between sEEG- and ECOG-specific on-off distributions in Subject 1.

In an additional exploratory analysis, different parametric functions were tested for goodness of fit in estimating the spectral power distributions of the headset-on neural signals. To do this, the chi-squared goodness of fit ( $\mathrm{g}$ ) metric was calculated between actual data and a given parametric estimate of the distribution. Although only the nonparametric kernel estimate represented the actual data with statistically significant goodness of fit $(\mathrm{p}=0.9553, \mathrm{~g}=0.6672)$, some parametric fits demonstrated moderately good fit, including exponential $(p=0.0017)$, Pareto $(p=0.0033)$, Log-Normal $(p=0.022)$, Birnbaum-Saunders $(p=0.0310)$, and Inverse Gaussian $(p=0.06)$ parametric distributions.

\subsubsection{Exploratory Analyses: Distribution Fits \& Regression}

Bandpower ratios were calculated to evaluate the magnitude of line noise or high frequency broadband noise power between different recording conditions (on vs off) and preprocessing steps (raw vs car).

$$
\text { Noise Ratio }=\frac{1}{n} \sum_{i=1}^{n}\left(\frac{P_{o n, i}}{P_{o f f, i}}\right), \text { Power } \sim \frac{V^{2}}{\sqrt{H z}}
$$

Scatter plots of line noise bandpower and noise ratio vs. electrode number as an ordinal label were also made in order to visualize potential trends in the bandpower (See Fig. 6). Trends were further explored using a linear regression of noise band power onto sEEG contact number as a proxy for implant depth. In intracranial implants, channel 1 is the furthest from the wire bundle leaving the skull, making electrode number a rough proxy for depth from cortex, with the higher numerical labels corresponding to electrodes nearer the cortex and lower numerical labels corresponding to electrodes at greater depths. In Subject 1, cortical ECoG electrodes were coded to have a label of 17 , which is one greater than the largest sEEG label (16). To measure the effect size of the regression coefficient, 1000 shuffled label permutations of the regression were completed and the average regression coefficient and intercept from these permutations was used to define the null regression. From this, a $\Delta \mathrm{r} 2$ value was determined.

A regression was implemented to explore the depth effect of the VR headset. Fig. 6 depicts an example regression result. In this splitaxis plot, the left y-axis is the average band power from $57-63 \mathrm{~Hz}$, and the right axis is the noise ratio. The $\mathrm{x}$-axis is the 1-16 electrode number indicative of electrode depth. The scatterplot dots in blue and red depict the band power distributions of the HMD On and HMD Off conditions. The regression magnitude was compared against a randomized null distribution, yielding a $\Delta \mathrm{r} 2$ value between the ratio regression and null (permutation) regression. It can be interpreted as an effect size of the correlation.

Regressions were completed for headset on and off, raw and car, for line noise or noise floor bandpower along electrode number. Fig. 6 plots the In Subject 1, the raw signal line noise bandpower correlates with depth with headset on $(\mathrm{r}=0.0985, \mathrm{p}=0.000054, \Delta \mathrm{r} 2$ on-null $=$ $0.00046)$, with headset off $(\mathrm{r}=0.055, \mathrm{p}=0.011, \Delta \mathrm{r} 2 \mathrm{on}-\mathrm{null}=$ $0.00032)$, and with headset on after CAR $(r=0.052, p=0.0136$, $\Delta \mathrm{r} 2$ on-null $=0.00026)$. High-frequency noise floor regresses in the headset on $(\mathrm{r}=0.087, \mathrm{p}=0.000026, \Delta \mathrm{r} 2 \mathrm{on}$-null $=0.00025, \Delta \mathrm{r} 2 \mathrm{on}$-off $=0.000013, \Delta \mathrm{r} 2$ on-CAR $=0.000058)$, off $(\mathrm{r}=0.092, \mathrm{p}=0.00000017$, $\Delta \mathrm{r} 2$ on-null $=0.00024)$, and on CAR $(\mathrm{r}=0.063, \mathrm{p}=0.00011)$.

In Subject 2, only raw signal line noise bandpower with the headset off correlates with depth $(\mathrm{r}=-0.150, \mathrm{p}=0.0011, \Delta \mathrm{r} 2$ off-null $=0.0089$,

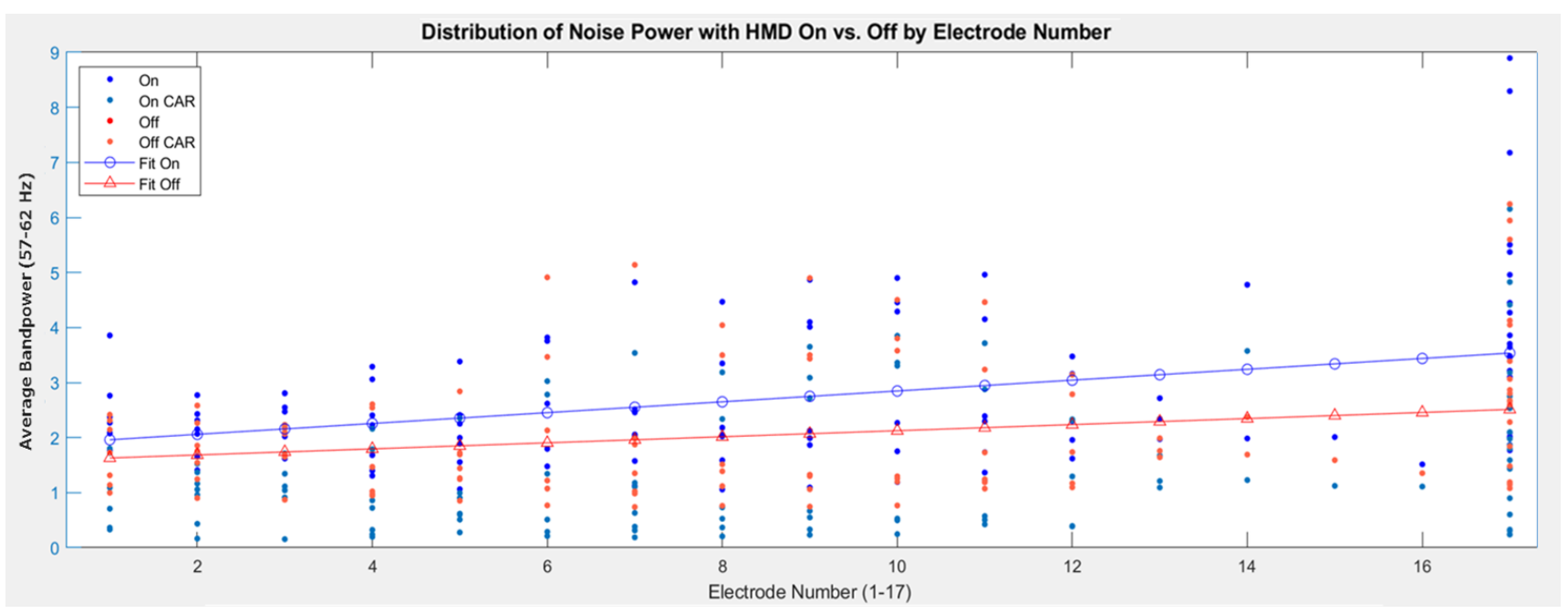

Fig. 6. A scatterplot of bandpower by electrode and line plot of the regression results for Subject 1. On the $y$-axis is bandpower for line noise $(57-62 \mathrm{~Hz})$. Values from both raw and CAR signals in the headset on and off conditions are plotted (blue shades for On and red shades for Off). The $x$-axis encodes electrode number with SEEG (1:16) and ECoG electrodes (coded as 17). The plotted regression lines for the raw data visualize the relationship between high-frequency noise and electrode number, a proxy for implant depth with 1 being the "deepest" electrode and 17 being a cortical or "surface" electrode. The high-frequency noise floor was found to significantly correlate with electrode number in both the headset on condition (blue line, $r=0.0985, p=0.000054, \Delta r 2$ on-null $=0.00046$ ) and off condition (red line, $r=0.055, p=0.011, \Delta r 20 n-n u l l=$ 0.00032), though only the headset on regression was preserved through CAR $(r=0.052, p=0.0136, \Delta r 2 o n-n u l l=0.00026)$. 


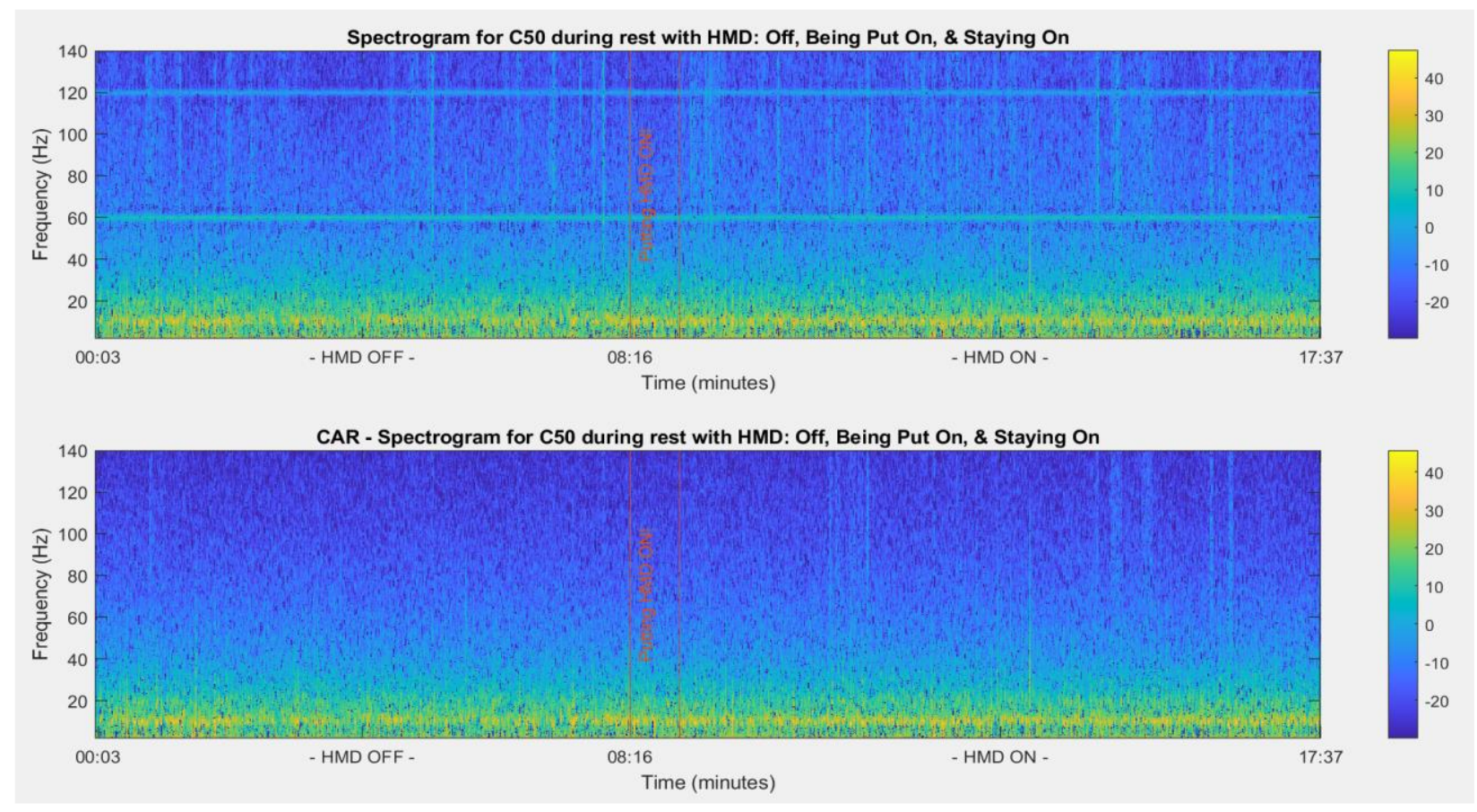

Fig. 7. Spectrograms depicts the spectral power content in $1 \mathrm{~Hz}$ frequency bands from $1-140 \mathrm{~Hz}$, with 1 second temporal resolution across a recording over 3 minutes in length in which the VR headset is put on. The left portion of these spectrograms depict a headset off condition, the segment between the red lines during headset donning, and the right portion of the spectrograms depict a headset on condition. The top plot is of raw signal and the bottom plot is of the same signal following CAR. Data presented were from an electrode localized to Brodmann Area 6 (BA 6) in Subject 2.

$\Delta \mathrm{r} 2 \mathrm{off}-\mathrm{on}=0.0145, \Delta \mathrm{r} 2 \mathrm{raw}-\mathrm{CAR}=0.010)$ and raw signal noise floor bandpower with headset off $(\mathrm{r}=-0.056, \mathrm{p}=0.001, \Delta \mathrm{r} 2 \mathrm{off}-\mathrm{null}=0.0092$, $\Delta \mathrm{r} 2$ off-on $=0.0140, \Delta \mathrm{r} 2$ off-CAR $=0.0098)$.

\subsubsection{Spectral Changes While Donning \& Doffing}

Lastly, in Subject 2, continuous neural recordings during headset positioning (donning or doffing) were identified. To visualize neural signals changed during headset positioning, time-frequency spectrograms were built by the modified Welch method (with $80 \%$ overlap and reassigned center frequencies, $1 \mathrm{sec}$ time resolution, 2$140 \mathrm{~Hz})$.

The stacked spectrograms of Fig. 7 each represent the same epoch of time, during which Subject 2 was at rest and not wearing the VR headset, then donning the headset with assistance, and then sitting in a virtual "waiting room" depiction of a dark sky at dusk. This length of time was selected to allow for visual evaluation of spectral power under the HMD Off, transition, and HMD On conditions. Visual comparison of the left (HMD Off) vs. right (HMD On) halves of each spectrogram shows brief time periods of increased high-frequency noise more frequently in the HMD On than Off condition. These brief periods are seen as vertical striping in the higher frequencies of the plot.

\section{Discussion}

Virtual reality behavioral task design represents a new and emerging platform for human neuroscientific research [7-10]. In this work, we quantified the impact of a VR research platform implementation on electrophysiological signal quality and fidelity specifically from intracranial recordings. This work represents, to the best of our knowledge, the first examination and implementation of an immersive VR task for patients implanted with intracranial electrodes in an inpatient setting.
In our noise analysis, we explored the impact of electrical noise from the HMD and demonstrated strong phase synchronization of headset-induced noise across implanted electrodes and efficient removal with common average referencing (CAR). Wearing the headset increased line noise effects and decreased the high-frequency broadband noise power, but these headset impacts were markedly reduced by CAR. In Subject 1, the regression analysis revealed a significant relationship between line noise power and electrode number in both headset on and off conditions, though the slope was about twice as steep with the headset on. The positive slope indicated that higher numbered or more cortical electrodes recorded signal with greater line noise. The regression analysis also demonstrated that CAR redistributed line noise power and flattened this linear relationship. Collectively, these results suggest that while the headset increases line noise in the neural recordings, this increase is readily addressed by a simple CAR preprocessing step.

Our noise analysis also concluded that wearing the HMD decreased the high-frequency broadband noise floor. In agreement, the regression analysis for Subject 2 demonstrated a significant relationship between increasing noise floor and electrode depth (lower electrode numbers) only when the headset was off. This may indicate that the VR headset does not introduce additional high-frequency noise or significantly increase the noise floor of neural recordings, but rather, that it may somehow be protective against penetrating highfrequency noise in neural recordings.

In this paper, we also discussed successful approaches and considerations for immersive VR research in the in-patient, invasive neuromonitoring patient population. This work represents, to the best of our knowledge, the first implementation of an immersive VR task for patients implanted with intracranial electrodes in an inpatient setting. We discussed VR hardware selection, room setup, and patient interaction. We also sought to address concerns about VR skill acquisition in VR-naïve populations, and demonstrated rapid VR 
immersion, task engagement, and genuine enthusiasm from both patients for VR tasks. Although a small sample size, our first two subjects not only well-tolerated their VR experiences but were enthusiastic to continue. We hope this allays concerns about HMD discomfort with this postoperative intracranial patient population, VR hesitancy, and the potential impact of VRS.

\section{Conclusion}

Virtual reality tasks were well-tolerated in two inpatient intracranial recording subjects who completed over 94 minutes of cumulative time in v.r. The VR headset was found to introduce phaselocked line noise and intermittent broadband high frequency noise. Both seemed addressable by common average and common median referencing. This work represents, to the best of our knowledge, the first implementation of an immersive VR task for patients implanted with intracranial electrodes in an in-patient setting.

\section{ACKNOWLEDGMENTS}

We would like to thank Dr. Alexander Doud for clinical data retrieval. We would also like to gratefully acknowledge the patients who participate in this research. Our work is supported by the National Science Foundation (EEC-1028725) and the UW Reality Lab which draws funding from Facebook, Google, Futurewei, and Amazon. The content is solely responsibility of the authors and does not necessarily represent the official views of any funding agency.

\section{References}

[1] D. J. Caldwell, J. G. Ojemann, and R. P. N. Rao, "Direct Electrical Stimulation in Electrocorticographic Brain-Computer Interfaces: Enabling Technologies for Input to Cortex," Front. Neurosci., vol. 13, p. 804, Aug. 2019.

[2] G. Simić and P. R. Hof, "In search of the definitive Brodmann's map of cortical areas in human," J. Comp. Neurol., vol. 523, no. 1, pp. 5-14, Jan. 2015.

[3] J. A. Cronin et al., "Task-Specific Somatosensory Feedback via Cortical Stimulation in Humans," IEEE Trans. Haptics, vol. 9, no. 4, pp. 515522, Oct. 2016.

[4] P. Kourtesis, S. Collina, L. A. A. Doumas, and S. E. MacPherson, "Technological Competence Is a Precondition for Effective Implementation of Virtual Reality Head Mounted Displays in Human Neuroscience: A Technological Review and Meta-Analysis," Front. Hum. Neurosci., vol. 13, p. 342, Oct. 2019.

[5] P. Kourtesis, D. Korre, S. Collina, L. A. A. Doumas, and S. E. MacPherson, "Guidelines for the Development of Immersive Virtual Reality Software for Cognitive Neuroscience and Neuropsychology: The Development of Virtual Reality Everyday Assessment Lab (VR-EAL), a Neuropsychological Test Battery in Immersive Virtual Reality," Frontiers in Computer Science, vol. 1, p. 12, 2020.

[6] G. Li et al., "Optimal referencing for stereo-electroencephalographic (SEEG) recordings," Neuroimage, vol. 183, pp. 327-335, Dec. 2018.

[7] G. Beraldo, A. Suppiej, C. Forest, L. Tonin, and E. Menegatti, "BrainComputer Interface for children: state-of-the-art and challenges*," in 2020 IEEE International Conference on Systems, Man, and Cybernetics (SMC), Oct. 2020, pp. 24-29.

[8] A. Ramirez-Zamora et al., "Proceedings of the Seventh Annual Deep Brain Stimulation Think Tank: Advances in Neurophysiology, Adaptive DBS, Virtual Reality, Neuroethics and Technology," Front. Hum. Neurosci., vol. 14, p. 54, 2020.

[9] D. D. Georgiev, I. Georgieva, Z. Gong, V. Nanjappan, and G. V. Georgiev, "Virtual Reality for Neurorehabilitation and Cognitive Enhancement," Brain Sci, vol. 11, no. 2, Feb. 2021, doi: 10.3390/brainsci11020221.

[10] U. Topalovic et al., "Wireless Programmable Recording and Stimulation of Deep Brain Activity in Freely Moving Humans," Neuron, vol. 108, no. 2, pp. 322-334.e9, Oct. 2020. 